\title{
Capnometry in the Assessment of Pulmonary Ventilation in Patients with Bronchial Asthma
}

\author{
Yurii Feshchenko ${ }^{1,}$, , Liudmyla Iashyna ${ }^{2}$, Svitlana Opimakh², Maryna Polianska ${ }^{2}$, \\ Viktoria Ignatieva $^{2}$, Svitlana Moskalenko², Galyna Gumenuk ${ }^{2}$, Inna Zvol' ${ }^{2}$, Nataliia Vlasova ${ }^{2}$, \\ Liudmyla Halai ${ }^{2}$ \\ ${ }^{1}$ Department of Pulmonology, Therapy and Clinical Pharmacology of Lung Diseases, National Institute of Phthisiology and Pulmonology, \\ Named After Feophil Gavrilovich Yanovskiy NAMS of Ukraine, Kiev, Ukraine \\ ${ }^{2}$ Department of Diagnostic, Therapy and Clinical Pharmacology of Lung Diseases, National Institute of Phthisiology and Pulmonology, \\ Named After Feophil Gavrilovich Yanovskiy NAMS of Ukraine, Kiev, Ukraine
}

\section{Email address:}

diagnost@ifp.kiev.ua (Y. Feshchenko), iashyna@ukr.net (L. Iashyna), sveta_infodoc@ukr.net (S. Opimakh),

r_polianma@ukr.net (M. Polianska)

${ }^{*}$ Corresponding author

\section{To cite this article:}

Yurii Feshchenko, Liudmyla Iashyna, Svitlana Opimakh, Maryna Polianska, Viktoria Ignatieva, Svitlana Moskalenko, Galyna Gumenuk, Inna Zvol, Nataliia Vlasova, Liudmyla Halai. Capnometry in the Assessment of Pulmonary Ventilation in Patients with Bronchial Asthma. American Journal of Internal Medicine. Special Issue: New Approaches to Manage Difficult-to-Control, Severe Asthma.

Vol. 8, No. 1, 2020, pp. 14-18. doi: 10.11648/j.ajim.20200801.13

Received: December 16, 2019; Accepted: January 3, 2020; Published: January 13, 2020

\begin{abstract}
Background: Bronchial obstruction as characteristic feature of bronchial asthma (BA) can lead to gas exchange disorders. The distinction of asthma is that even in patients without clinical symptoms and almost normal spirometry parameters may have been significant gas exchange disorders including that due alveolar ventilation reducing. This study aimed to investigate lung ventilation pecularities in patients with BA. Results: Among patients with uncontrolled course of $40 \%$ have a smoking history with index $(25,8 \pm 8,8)$ pack-years. In patients with uncontrolled asthma "dead" space ventilation volume is $(211 \pm 8) \mathrm{ml}$, and part of the "dead" space of the tidal volume - $(30,5 \pm 1,5) \%$, which is statistically significantly greater than that rate for patients with controlled asthma - $(176 \pm 11) \mathrm{ml}$ and $(24,6 \pm 0,5) \%$, respectively, $\mathrm{p}<$ 0.05. Alveolar ventilation in patients with uncontrolled disease course is lower, namely $(7,3 \pm 0,4) 1 / \mathrm{min}$. than when controlled asthma - $(8,3 \pm 0,2), \mathrm{p}<0.05$. Conclusions: Lung ventilation parameters in patients with asthma without exacerbation in general slightly different from those in healthy subjects, but the effectiveness of alveolar ventilation is reduced by $14 \%$ in uncontrolled asthma due to high waste "dead" space ventilation.
\end{abstract}

Keywords: Bronchial Asthma, Lung Ventilation, Alveolar Ventilation

\section{Introduction}

Asthma is a heterogeneous disease, usually characterized by chronic airway inflammation. It is defined by the history of respiratory symptoms such as wheeze, shortness of breath, chest tightness and cough that vary over time and in intensity, together with variable expiratory airflow limitation. Both symptoms and airflow limitation characteristically vary over time and in intensity [1].

Even when symptoms are episodic, the inflammation in asthma persists constantly and occurs in all clinical forms of asthma in all age groups of patients. Inflammation alters the structure of the airways, defined as airway remodeling [2].

Limitation of bronchial permeability leads to impairment is gas exchange, especially through ventilation-perfusion imbalance. Even in patients without clinical symptoms and almost normal spirometry parameters significant disturbances in gas exchange may be present [3].

To determine the presence, severity of bronchial obstruction and its reversibility today applies pulmonary function testing - spiromentry, bodyplethysmography (allow to detect lung hyperinflation [4, 5]). But spirometry and 
bodyplethysmography did not allow to determine, whether alveolar ventilation is effective in asthma patients.

The preferred exact method of evaluating lung ventilation effectiveness is analysis of arterial blood gases. But it is invasive and causes some inconvenience to the patient, especially in patients with poor vascular access. On of the requirements for correct blood sampling is a steady state without changes in ventilation parameters. For patients with bronchial obstruction this time is 20-30 minutes. Blood is alive tissue, and cellular metabolism (absorption of oxygen and production of $\mathrm{CO}_{2}$ ) lasts after the moment of sampling till the beginning of analysis, what distorts the result. At the same time air exposure reduces the $\mathrm{CO}_{2}$ level in the samples. That's why blood gas analysis should be carried out immediately [6]. Up-to-date in Ukraine analysis of arterial blood gases is not always available for patients with asthma due to the high cost of equipment and supplies.

The possible way to assess the effectiveness of pulmonary ventilation is the study of alveolar ventilation. Alveolar ventilation and minute volume of breathing are not identical values. Alveolar ventilation - is the volume of air that not only reaches the alveoli, but also takes part in gas exchange. The factual minute ventilation, in addition to alveolar ventilation, includes ventilation of airways, which are not involved in gas exchange (anatomical "dead" space) and alveoli that are not properly perfused (alveolar "dead" space). The difference in minute and alveolar ventilation - is physiological "dead" space (VD, dead space), which is the sum of anatomical and alveolar "dead" space. The higher becomes physiological "dead" space volume as part of minute ventilation, the smaller will be the alveolar ventilation with the development of gas exchange disorders [7-9].

VD/VT ratio (the part of the "dead" space of the tidal volume) - is a sensitive indicator of gas exchange, reflecting concordance between ventilation and perfusion at rest as well as during exercise. In patients with respiratory diseases $\mathrm{VD} / \mathrm{VT}$ increase primarily due to non-uniformity of ventilation and perfusion in the lung, while in patients with heart failure and pulmonary vascular disease VD/VT impaires primarily due to changes in blood flow with preserved ventilation [10]. We believe that the determination of VD/VT can be a useful tool for assessment of alveolar ventilation in patients with asthma.

In asthma the imbalance of alveolar ventilation ( $\mathrm{Va}$ ) and circulation $(\mathrm{Q})$, one of the key factors maintaining normal concentrations of arterial blood gases take place. An objective assessment of $\mathrm{Va} / \mathrm{Q}$ balance is the method of multiple inert gas elimination technique (MIGET), wherein a mixture of six inert gases with different solubility administered intravenously to the patient. After 30 minutes (after equalization of gases concentrations in the body) conducts the analysis of the content of inert gases in the exhaled air, in the arterial blood and blood from the pulmonary artery. The results of the mathematical analysis of received data describe the distribution of ventilation and perfusion [11]. The main drawback of this method, we consider the high complexity of performance and its unavailability in Ukraine.

We studied the possibility of capnometry in evaluating of pulmonary ventilation in patients with asthma. Capnometry is measurement and digital display of concentration or partial pressure of carbon dioxide in the air that patient inhales or exhales during the respiratory cycle [12]. By integrating capnometer and pneumotachometers in one device and the unique opportunity of capnometry to calculate $\mathrm{CO}_{2}$-free air portions during exhalation as the volume of the "dead" space and its part in the tidal volume makes it possible to evaluate the efficiency of alveolar ventilation [13].

The aim of our study was investigate features of lung ventilation in patients with BA. For this purpose the following tasks were decided:

a. To investigate parameters of lung ventilation in patients with BA and healthy subjects;

b. To compare lung ventilation parameters in patients with BA depending on disease control.

\section{Materials and Methods}

This work was financed from the state budget of Ukraine.

The study was coordinated with the local Medical Ethics Committee of the NIPhP NAMS, participants were familiarized with the study protocol and signed an informed consent form before the beginning of study procedures.

Inclusion criteria for asthma patients - male and female, $\geq$ 18 years, with reversibility of forced expiratory volume at 1 second $\left(\mathrm{FEV}_{1}\right) \geq 12 \%$ (and $\geq 200 \mathrm{ml}$ ) after inhalation of 400 mcg of short acting $\beta_{2}$-agonists, free of exacerbation, familiarizied with the study protocol and signed informed consent to participate in the study, able to understand and perform diagnostic procedures.

Inclusion criteria for healthy individuals - male and female, $\geq 18$ years, absence of the history of respiratory diseases, normal physical examination, with $\mathrm{FEV}_{1}>80 \%$ of the predicted value and $\mathrm{FEV}_{1} / \mathrm{FVC}$ ratio $>70 \%$, familiarizied with the study protocol and signed informed consent to participate in the study, able to understand and perform diagnostic procedures.

Exclusion criteria - other, than asthma, respiratory diseases and severe uncontrolled progressive chronic diseases that can affect the results of investigation, mental disorders.

The diagnosis of asthma as defined by GINA criteria at least 1 year before Visit 1 .

30 asthma patients (11 male and 19 female), mean age $(57,3 \pm 2,3)$ years and mean $\mathrm{FEV}_{1}(72,3 \pm 1,8) \%$ and 35 healthy individuals (19 male and 16 female), mean age $(48,0$ $\pm 2,4)$ years and mean $\operatorname{FEV}_{1}(99,1 \pm 1,5) \%$ took part in this study.

Asthma Control Test (ACT) was performed in asthma patients.

Capnometry was conducted for all participants ("Oxycon Pro", "Cardinal Health" (Germany), with the evaluated of following parameters:

a. the "dead" space volume, $\mathrm{ml}(\mathrm{VD}, \mathrm{ml})$;

b. part of the "dead" space of the tidal volume, $\%$ (VD\% 


\section{VT)}

c. volume ventilation liters per minute $\left(\mathrm{V}^{\prime} \mathrm{E}, \mathrm{L} / \mathrm{min}\right)$,

d. alveolar ventilation volume ( $\left.\mathrm{V}^{\prime} \mathrm{E}, \mathrm{L} / \mathrm{min}\right)$.

Prior to the test the essence of the procedure explained to the patient. The procedure conducted in the sitting position. The patient was breathing ambient air for 5 minutes through a mouthpiece with a nose clip (to entire the inhaled or exhaled flow passed through the analyzer). Then within 3 minutes data of the gas analysis were recorded. The device displays the average of the concentration or partial pressure of $\mathrm{O}_{2}$ of every four successive respiratory cycles.

Data collection and mathematical processing were carried out by licensing software products included in the package Microsoft Office Professional 2007 license Russian Academic OPEN No Level № 43437596. Statistical analysis was performed using mathematical and statistical features MS Excel. The parameters studied in this work were evaluated by determining the mean (M), the mean error (m), reliability $(t)$, the level of significance $(p)$.

\section{Results and Discussion}

Capnometry was analyzed for all 65 study participants.

To resolve the first task of our work we have found that the parameters of pulmonary ventilation in patients with asthma in general slightly different from those in healthy subjects. Thus, the average minute ventilation accounted $(10,5 \pm 0,3)$ $1 /$ min in healthy subjects vs $(10,7 \pm 0,4) 1 /$ min in BA patients (Figure 1). As for "dead" space ventilation, it was on average higher in patients with asthma - $(173 \pm 8) \mathrm{ml}$, compare to healthy subjects $-(189 \pm 8) \mathrm{ml}$, but without statistically significant difference. Higher, but without statistically significant difference, was the part of the "dead" space of the tidal volume in BA patients $-(27,4 \pm 1,3) \%$, while in healthy individuals it is $(25,2 \pm 0,9) \%$.
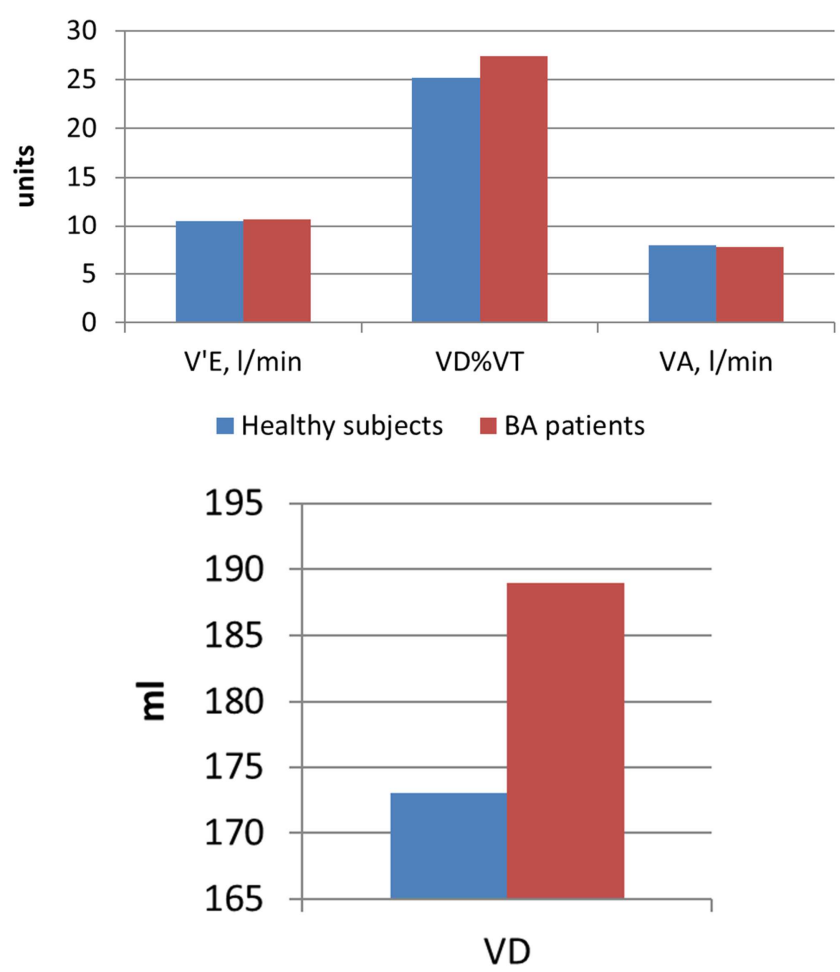

Note: without statistically significant difference.

Figure 1. Lung ventilation indicators in healthy subjects and in BA patients.

According ACT asthma patients were divided into 2 groups: controlled (ACT $>20$ points) and uncontrolled (ACT $<20$ points). Clinical characteristics of patients are presented in table 1 .

Table 1. Clinical characteristics of $B A(n=30)$ patients depending on disease control $(M \pm m)$.

\begin{tabular}{lll}
\hline Indicators & Controlled BA patients, $\mathbf{n}=\mathbf{1 0}$ & Uncontrolled BA patients $\mathbf{n}=\mathbf{2 0}$ \\
\hline Male $(\mathrm{n}, \%)$ & $3(30 \%)$ & $8(40 \%)$ \\
Female $(\mathrm{n}, \%)$ & $7(70 \%)$ & $12(60 \%)$ \\
Age, years & $59,7 \pm 2,7$ & $56,1 \pm 3,2$ \\
ACT, points & $21,6 \pm 0,4$ & $11,8 \pm 0,6^{*}$ \\
Mean $\mathrm{FEV}_{1}, \%$ & $73,8 \pm 4,2$ & $71,5 \pm 1,9$ \\
\hline
\end{tabular}

Note: $*$ - statistically reliable difference of indicators between groups, $\mathrm{p}<0,01$.

Smoking status of patients with asthma (Figure 2.):

a. Among patients with controlled asthma $90 \%$ were no smokers. The single smoker in the group has smoking history of 5 pack-years.

b. Among patients with uncontrolled asthma $40 \%$ had mean smoking history $(25,8 \pm 8,8)$ pack-years (within 1,5 - 40 (!) pack-years). The data correspond to a recognized position that smoking contributes to more severe, uncontrolled asthma course. Also, smoking decreases the effectiveness of anti-inflammatory therapy, which also contributes to the loss of control of the disease [14].

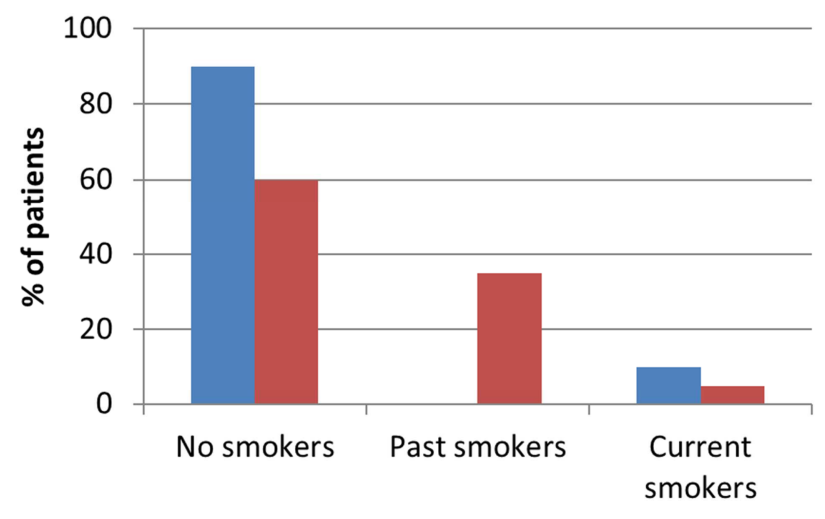

Controlled BA patients Uncontrolled BA patients 


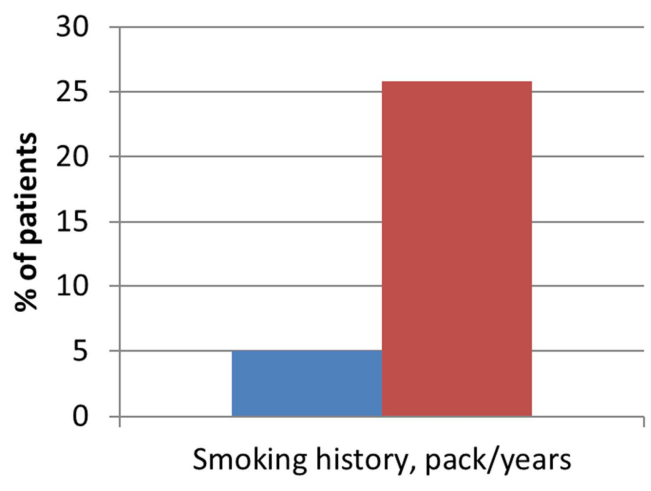

Figure 2. Smoking history in asthma patients.

Note: no correct mathematical bases for determining statistically significant difference between indexes of the observation groups.

As to the second objective of our work - it was found that the minute ventilation didn't differ significantly in controlled and uncontrolled asthma patients (Figure 3).

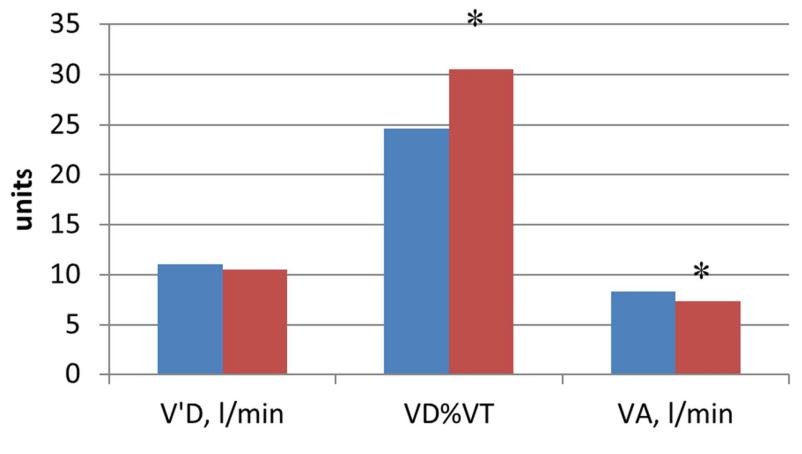

- Controlled BA patients Uncontrolled BA patients

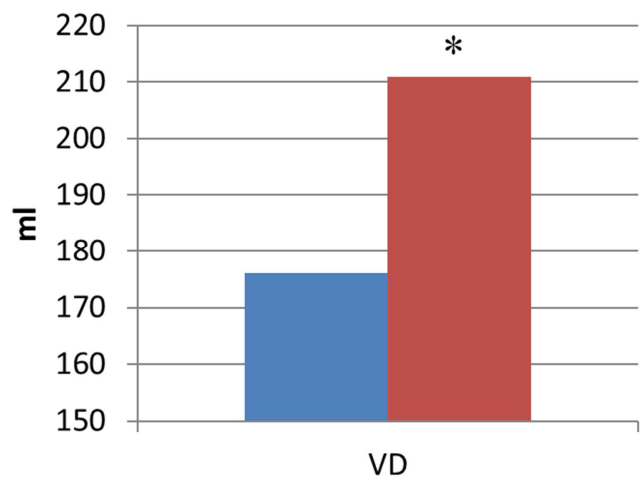

Figure 3. Minute ventilation in asthma patients.

But in patients with uncontrolled asthma "dead" space ventilation volume was $(211 \pm 8) \mathrm{ml}$, which is statistically significantly higher than the rate for patients with controlled course $-(176 \pm 11) \mathrm{ml}, \mathrm{p}<0,05$. Thus, the part of the "dead" space of the tidal volume in uncontrolled asthma patients is $(30,5 \pm 1,5) \%$, whereas in subjects with controlled asthma it is statistically reliable smaller $-(24,6 \pm 0,5) \%, p<0,05$. It's obvious that with an equal minute ventilation in patients with high VD\% VT alveolar ventilation will be lower - $(7,3 \pm 0,7)$ $1 /$ min in patients with uncontrolled asthma, that is $14 \%$ and significantly lower vs $(8,3 \pm 0,2), \mathrm{p}<0,05$ in controlled asthma patients.

We explain our findings that violations of pulmonary ventilation in patients with uncontrolled asthma may be associated with impairment of small airways and with the development of lung hyperinflation. Thus, according to bodyplethysmography, the value of the residual lung volume in controlled asthma patients is nearly normal $(121,3 \pm 10,5) \%$ pred, whereas in uncontrolled it is much greater - $(132,6 \pm 8,3)$ (standard normal values of lung volumes is a range of 80 to $120 \%$ of the predicted values [5]).

We got a question: whether there is a natural development of lung hyperinflation in patients with a mean $\mathrm{FEV}_{1}(71,5 \pm$ $1,9) \%$ pred? According to the literature this may be true trend, because we found the evidence that formation of air "traps" is observed not only at a moderate, but also for mild asthma [15]. In the study of the concentration of nitric oxide in the exhaled air, it was found that in the stable asthma detected inflammatory lesions not only in distal airways but also in lung acinar areas [16].

There is evidence that inflammation in the distal airways is more intense in severe and uncontrolled asthma course. Formation of pulmonary hyperinflation in patients with asthma is associated with the defeat of the distal airways [17].

Thus, the patients with uncontrolled asthma are characterized by: a history of smoking, development of lung hyperinflation with an increase the part of the "dead" space in the tidal volume and decrease of efficiency of alveolar ventilation.

\section{Conclusions}

a. Lung ventilation parameters in patients with stable asthma (beyond exacerbation) in general slightly different from those in healthy subjects;

b. The effectiveness of alveolar ventilation is reduced by $14 \%$ in uncontrolled asthma due to high waste "dead" space ventilation.

\section{References}

[1] GINA Report, Global Strategy for Asthma Management and Prevention, 2019. URL: https://ginasthma.org/gina-reports/ (last accessed 08.07.2018).

[2] B. N. Lambrecht, H. Hammad, J. V. Fahy (2019). The Cytokines of Asthma. Immunity. 50 (4): 975-991. doi: 10.1016/j.immuni.2019.03.018.

[3] Lui, J. K. \& Lutchen, K. R. Clin Trans Med (2017) 6: 29. https://doi.org/10.1186/s40169-017-0159-0.

[4] Brusasco V., Barisione G. (2019) Tests of Lung Function: Physiological Principles and Interpretation. In: Cogo A., Bonini M., Onorati P. (eds) Exercise and Sports Pulmonology. Springer, Cham.

[5] Yu. I. Feschenko, L. A. Iashyna, K. V. Nazarenko, S. M. Moskalenko, S. G. Opimakh, N. M. Musienko (2018). Diagnostics of hyperinflation and emphysema in patients with asthma and copd overlap on the primary and specialized levels of medical care. Ukr. Pulmonol. J. 1: 7-13. 
[6] Davis M. D., Walsh B. K., Sittig S. E., Restrepo R. D. (2013) AARC clinical practice guideline: blood gas analysis and hemoximetry. Respir Care. 58: 1694-703.

[7] Quinn M, Rizzo A. StatPearls [Internet]. StatPearls Publishing; Treasure Island (FL): Dec 6, 2018. Anatomy, Anatomic Dead Space.

[8] Intagliata S, Rizzo A, Gossman WG. StatPearls [Internet]. StatPearls Publishing; Treasure Island (FL): May 15, 2019. Physiology, Lung Dead Space.

[9] Nieves A, Cozzo A, Kosoff Z, et al. (2019). 3D airway model to assess airway dead space. Archives of Disease in Childhood - Fetal and Neonatal Edition. 104: F321-F323.

[10] Yu. I. Feschenko, L. O. Iashyna, K. V. Nazarenko, S. G. Opimakh (2017). Assessment of alveolar ventilation in patients with asthma, COPD and their combination. www.umj.com.ua, 5 (121) - IX/X 2017. URL: https://www.umj.com.ua/wp/wpcontent/uploads/2017/09/4575.pdf?upload $=$.

[11] Michael Jaeger J., Titus B. J., Blank R. S. (2019) Essential Anatomy and Physiology of the Respiratory System and the Pulmonary Circulation. In: Slinger P. (eds) Principles and Practice of Anesthesia for Thoracic Surgery. Springer, Cham.
[12] Singh S., Kodali B. S. (2017) Volume capnography: A narrative review. Indian Anaesth Forum. 18: 33-8.

[13] Yu. I. Feschenko, L. A. Iashyna, K. V. Nazarenko, S. G. Opimakh (2017). Exercise testing influence on pulmonary ventilation parameters in patients with bronchial asthma, chronic obstructive pulmonary disease and their combination. Asthma and allergy. 2: 7-12.

[14] Chatkin, J. M., \& Dullius, C. R. (2016). The management of asthmatic smokers. Asthma research and practice, 2, 10. doi: 10.1186/s40733-016-0025-7.

[15] J.-M. Tunon-de-Lara, F. Laurent, V. Giraud, et al. (2007) Air trapping in mild and moderate asthma: Effect of inhaled corticosteroids. Journal of Allergy and Clinical Immunology. 119 (3): 583-590.

[16] S. Verbanck, D. Schuermans, W. Vincken (2010). Inflammation and airway function in the lung periphery of patients with stable asthma. J. Allergy Clin Immunol. 125: 611-616.

[17] Yu. Feschenko, L. Iashyna, K. Nazarenko and Svitlana Opimakh (2018) Factors of formation of small airways obstruction and lung hyperinflation in patients with combined pathology of asthma and chronic obstructive pulmonary disease. The Pharma Innovation Journal. 7 (1): 74-78. 